\title{
Étudier les représentations des prisons au musée, entre sémiotique des pratiques et analyse des interactions
}

\author{
Julien Thiburce ${ }^{1}$, Justine Lascar ${ }^{1}$, Isabel Colón de Carvajal ${ }^{2 \mathrm{a}}$ \\ ${ }^{1}$ CNRS, UMR 5191 ICAR \\ ${ }^{2}$ ENS de Lyon, UMR 5191 ICAR
}

\begin{abstract}
Résumé. Les musées constituent le lieu de pratiques et l'objet d'études d'une diversité de disciplines en sciences sociales. De manière privilégiée, les recherches menées en muséographie portent sur la confrontation entre les dispositifs scénographiques et l'expérience des visiteurs, entre les médiations qui tendent à garantir la prise sur l'exposition et les actions des visiteurs par lesquelles s'ajustent des savoirs et des sensibilités. À partir d'une perspective en sciences du langage, on problématise la constitution de données audiovisuelles de visites guidées pour l'étude des représentations des prisons en contexte muséal, dans le cadre d'un projet en partenariat avec le MICR (Genève), le Musée des Confluences (Lyon) et le Mémorial national de la prison Montluc (Lyon). Après avoir présenté les questions qui se sont imposées dans la prise en compte des spécificités de ces trois sites, on argumente la pertinence d'une méthode transversale à la sémiotique des pratiques et à l'analyse des interactions pour le recours aux vidéos dans un matériau d'enquête plus vaste.
\end{abstract}

\begin{abstract}
Investigating representations of prisons in museum, between semiotics of practices and interactions analysis. Museums are the place of practices and the object of studies for a variety of disciplines in the social sciences. In a privileged way, the researches carried out in museography focuse on the confrontation between scenographic devices and the visitors experience, between the mediations that tend to guarantee control over the exhibition and the visitors' actions through which knowledge and sensitivities are adjusted. From a language sciences perspective, we problematize the audiovisual recording of guided tours for the study of representations of prisons in museums, as part of a research project with the Red Cross Museum (Geneva), the Musée des Confluences (Lyon) and the Montluc National Prison Memorial (Lyon). After presenting the questions that arose in considering the specificities of these three museum sites, we argue the relevance of a transversal method to the
\end{abstract}

\footnotetext{
a Adresses de correspondance : julien.thiburce@ens-lyon.fr et justine.lascar@ens-lyon.fr
} 
semiotics of practices and the interactions analysis for the use of video in a broader material.

\section{Une sémiotique des interactions en situation de visite guidée}

Les expositions en milieu muséal constituent le lieu de pratiques et l'objet d'études d'une diversité de disciplines en sciences humaines. De manière privilégiée, les recherches menées en muséographie, champ souvent inséré dans le cadre plus large des sciences de l'information et de la communication, portent sur la confrontation entre les dispositifs scénographiques et l'expérience des visiteurs. Ces recherches consistent notamment à observer les tensions entre les médiations par lesquelles les instances de production du parcours d'exposition cherchent à garantir la prise des visiteurs sur celui-ci et les conduites d'actions des visiteurs à travers lesquelles se réalisent des négociations entre des savoirs experts et des savoirs profanes. Dans un dialogue épistémologique avec une perspective communicationnelle des pratiques muséales, on identifie deux approches de manière spécifique: une approche qui porte sur les ajustements, les interprétations et les qualifications des pratiques culturelles en action (Jutant, 2011) et une approche centrée sur la dimension expérientielle des visites (Schmitt, 2018). La première, qui se situe dans le paradigme de l'ethnométhodologie telle qu'elle a été développée, entre autres, par Harold Garfinkel (1967) et par Harvey Sacks, Emmanuel Schegloff et Gail Jefferson (1974), vise à "pouvoir saisir à la fois ce qui est mobilisé et ce qui est produit dans le cours de l'interaction » (Jutant, $2011:$ 42). La deuxième approche explore " comment [les visiteurs] construisent du sens à travers un ensemble de mouvements, de perceptions, d'émotions, qui font ce qu'ils nomment eux-mêmes de l'«information »» (Schmitt, $2018: 95)^{1}$, se plaçant dans le paradigme énactionniste pour lequel l'élaboration d'un « micro-monde », ordinaire « succession de configurations changeantes qui surgissent et se dissipent » (Varela, [1996] 2004 : 60), implique des résistances de l'environnement.

Cette construction de sens à travers un ensemble de mouvement, de perception et d'émotions trouve des résonances dans le champ des sciences du langage où nous nous situons. Pour étudier les représentations des prisons en contexte muséal ${ }^{2}$ au sein de ce champ, nous élaborons un dialogue entre une sémiotique des pratiques (Fontanille, 2008), qui dresse une perspective modélisante des cours d'actions mobilisant des objets et des textes, et une sémiotique vive (Basso Fossali, 2008), qui pense le sens comme une écologie de sens qui profite des traductions continues entre la signification discursive et l'expérience. Aussi, en vue d'étudier les propres perceptions, interprétations et énonciations des participants aux visites guidées des différents sites où nous menons notre recherche, on ne peut que profiter des développements majeurs apportés par l'analyse des interactions d'inspiration ethnométhodologique (Mondada, 2008) et par l'analyse du discours qui étudie les dynamiques d'assomption, d'imputation et de revendication de points de vue à travers les phénomènes de prise en compte et de prise en charge énonciatives (Rabatel, 2017). Dans cet horizon épistémologique, la visite guidée, en tant qu'espace de co-construction et de négociation des expériences individuelles et collectives (Dufiet, 2012 ; Kreplak 2014 ; Traverso et Ravazzolo, 2016), semble un lieu privilégié pour enquêter sur les transformations des sensibilités et des connaissances dans le cours même des interactions. Ainsi, si le musée constitue l'espace d'implémentation d'un discours institutionnel (Goodman, 1984) auquel se confrontent les visiteurs et l'environnement d'une expérience de visite, il apparaîtrait fondamental d'étudier les tensions entre la visée de l'énonciation institutionnelle du parcours d'exposition et la saisie opérée à travers l'énonciation collaborative au cours de la situation de visite guidée. 


\subsection{La constitution et l'articulation des données d'un corpus complexe}

Dans l'approche adoptée, nous tentons de problématiser le rôle des pratiques langagières dans le déploiement d'une médiation en groupe, à partir d'une observation et d'une caractérisation des enchevêtrements entre perception et énonciation : ce qui est en train d'être dit sur ce qui est en train d'être perçu par les participants à une activité en public donne des indices pour pister les processus sémiosiques par lesquels des personnes font émerger un sens de leur interaction. On cherche ainsi à décrire comment la conduite de ces interactions est elle-même gérée par les coparticipants et comment les contenus thématiques, encyclopédiques et axiologiques sont traités collectivement. Pour étudier ces enchevêtrements, il nous faudra rendre compte des liens consubstantiels entre les dynamiques interactionnelles entre les personnes coprésentes et les interactions entre les personnes et leur environnement matériel. C'est ici même que s'affirme l'intérêt d'une approche transversale aux sciences du langage: si l'on s'intéresse aux dynamiques de gestion du sens de l'expérience à travers une énonciation en groupe, il semble dès lors nécessaire de prendre en compte et de décrire les mécanismes par lesquels des contenus référentiels et relationnels (Kerbrat-Orecchioni, 1992 ; De Stefani, 2012) se transforment.

À cette problématisation théorique, on couple une réflexion sur le plan méthodologique en se demandant quelles sont les méthodes déjà existantes sur lesquelles s'appuyer, celles à écarter et celles à développer selon une ingénierie de la recherche. Historiquement, les sciences du langage, notamment dans une approche lexicologique, ont constitué des systématisations à partir d'exemples forgés sur le fond d'une expérience personnelle et idiosyncrasique. Ceci n'est pas sans poser problème quant à la dimension sociale et culturelle des exemples mobilisés : le caractère grammatical et valide d'un terme, d'un énoncé ou d'une structure syntaxique est déterminé par l'expérience même des lexicologues en question. Ce type d'exemple a, petit à petit, cédé la place à des corpus d'attestation de pratiques langagières, en ayant recours à des discours déjà produits (par exemple, des textes littéraires, des discours médiatiques, des discours programmateurs) ou en constituant des enregistrements audio ou audiovisuels de diverses situations (de visites guidées, d'apéritifs entre amis, de consultations médicales, entre autres) ${ }^{3}$. Lorsqu'ils sont produits dans une approche écologique des activités socio-langagières - naturally occurring data (Lynch, 2002 ; Mondada, 2006 ; Traverso, 2012) -, ces enregistrements visent à préserver et à décrire les liens bilatéraux entre les pratiques enquêtées et l'environnement de l'interaction, sur le plan matériel comme sur le plan symbolique ${ }^{4}$.

Dans le contexte des pratiques muséales qui nous occupe ici, la constitution d'un corpus d'enregistrements audiovisuels de visites guidées s'avère donc indispensable, notamment pour retracer le développement progressif d'un contenu thématique et caractériser l'organisation séquentielle de la parole-en-interaction (Mondada, 2006, 2012 et 2014). La complexité de ce type de corpus se retrouve selon nous sur trois plans liés les uns aux autres :

- sur le plan technique, méthodologique et pratique, cette complexité relève de l'agencement, de l'amont vers l'aval, de la conception du dispositif de constitution des enregistrements, de la transcription et de l'annotation des données et de la densité de l'analyse dans une perspective linguistique et sémiotique ;

- sur le plan sémiotique, la complexité des données relève de l'emboîtement d'espaces énonciatifs, en ce que la mise en scène des paroles dans les musées implique une prise en compte des agencements entre un geste instaurateur, son implémentation dans un espace muséal et sa réélaboration sémantique dans le cours d'une visite (par exemple, des photos de cellules prises en prison sont articulées à d'autres objets de l'exposition et se trouvent réinterprétées in situ, dans les musées, par les visiteurs);

- sur le plan social et politique, c'est la dimension dialogique de la pratique de la recherche qui revêt une certaine complexité, où la question se pose aux chercheurs de la 
(re)prise en charge des voix et des points de vue des acteurs impliqués dans la gestion collective des représentations des prisons en contexte muséal (dont les institutions muséales, institutions carcérales, les institutions politiques, les personnes incarcérés, les publics).

Aussi, il est déterminant de savoir quel dispositif mobiliser ou développer en fonction des contraintes du terrain d'enquête, dans la mesure où (i) les questions de recherche et les choix concernant le dispositif de constitution de données se définissent mutuellement ; (ii) tous les dispositifs de constitution de données ne se valent pas $^{5}$.

\subsection{Baliser le terrain d'enquête : quelles dynamiques de muséalisation ?}

Dans notre projet de recherche PrisM (Prisons et Musées), la focalisation sur les publics de groupe s'est opérée de manière concertée avec la direction du développement culturel du Musée des Confluences, qui nous avait sollicité pour participer à une étude de la réception des publics de l'exposition temporaire et itinérante Prison. Notre observation des pratiques en situation de visite guidée s'affirme pertinente à la fois sur le plan interdisciplinaire (pour une mise en discussion des méthodes en sciences humaines) et sur le plan interprofessionnel (pour une mise en regard des approches des chercheurs et praticiens des musées).

Dans le cas de cette coproduction de l'exposition Prison, les prisons sont mises en discours dans des musées qui programment et produisent une diversité d'expositions thématiques par le biais de médiations tierces (objets de prisonniers, photographies, vidéos, encarts, entre autres). Cette première dynamique de production d'un discours muséal nous a alors poussés à travailler sur une deuxième dynamique, où un ancien espace carcéral se trouve reconverti en musée, en vue de proposer un discours sur la mémoire et l'histoire de ce lieu : c'est le cas du Mémorial national de la prison de Montluc que nous avons choisi pour troisième site d'observation. Une étude transversale de ces deux dynamiques de muséalisation paraît opportune pour caractériser "sur pièces » les (ré)appropriations du discours muséal par les participants aux interactions, les transformations des représentations socio-politiques sur les prisons au fil des visites et les tensions de l'expérience spatiale (Bossé, 2015) des prisons. Nous faisons en effet l'hypothèse que la documentation des visites guidées de ces différents sites nous donnera des indices pour observer et décrire des modalités de projection épistémique et affective différentes, propres à ces deux dynamiques de muséalisation, entre (i) le fait de les parcourir "à distance » à travers des médiations tierces qui tentent de faire entrer les visiteurs « en immersion » dans des espaces correctionnaires et (ii) le fait de parcourir une ancienne prison in situ en faisant émerger les stigmates des expériences passées sur un mode presque archéologique (le visiteur retrace l'inscription d'un.e ancien.ne détenu.e sur les murs).

Cette pertinence, identifiée en amont sur le plan épistémologique, a finalement rencontré l'intérêt des institutions muséales, sur les plans sociaux, culturels et éducatifs : le Musée des Confluences et le Mémorial national de la prison de Montluc avaient déjà conçu et programmé eux-mêmes un dispositif de médiation de visite "duo ». À destination de publics scolaires (lycées), ce type de visite se réalise dans la même journée sur ces deux sites lyonnais de manière à proposer une approche comparée des lieux pénitentiaires, comme on peut le lire sur le site internet du Musée des Confluences (http://www.museedesconfluences.fr/fr $)^{6}$.

Dans la conception et la pratique de notre méthode d'enquête, il convient alors de prendre en compte les spécificités de chaque site muséal et des activités qui s'y déploient, aussi bien sur le plan institutionnel, culturel et économique que sur le plan architectural et pratique. Nous présentons dans la prochaine section $(\S 2)$ le dispositif mobilisé en fonction des activités que nous suivons et documentons. Nous nous focalisons ensuite $(\S 3)$ de 
manière privilégiée sur une méthode développée dans notre recherche pour conserver un lien entre une perspective micro-analytique qui favorise l'étude de phénomènes interactionnels localement situés et une perspective macro-analytique qui cherche à avoir une prise sur le déroulement global d'une même visite et à décrire les continuités entre des visites, les unes par rapport aux autres.

\section{La visite guidée et ses différentes configurations}

Pour la première modalité de muséalisation, nous étudions des visites guidées qui se déroulent dans deux types de configuration. Dans une première configuration, on trouve des visites de l'exposition temporaire Prison d'environ $1 \mathrm{~h}$ auprès de publics scolaires et de publics non-scolaires. Dans un second type, les visites guidées de l'exposition temporaire durent environ 45 minutes et sont conduites par un médiateur dans le cadre d'ateliers webradios intitulés « Reporter au musée ", proposés à des publics (pré)adolescents (âgés de 10 à 15 ans), dont la visée est de sensibiliser les participants aux pratiques médiatiques et journalistiques en milieu muséal ${ }^{7}$. Pour le deuxième type de muséalisation, nous suivons des visites guidées du Mémorial national de la prison Montluc : lors des parcours que nous documentons, les participants peuvent accéder à l'aile des femmes habituellement fermée aux publics. Le dispositif de recueil que nous utilisons est le même pour les visites de l'exposition Prison et pour celles du Mémorial Montluc. Il diffère en revanche pour les ateliers "Reporter au musée ». Avant de pointer leurs différences sur le plan qualitatif, le tableau ci-dessous représente la durée respective de chacun des enregistrements constitués et leur durée totale, pour un aperçu quantitatif des données primaires qui constituent notre corpus de travail.

Tableau 1. Événements documentés et durée des enregistrements en date du 25 mars 2020

\begin{tabular}{|l|l|l|}
\hline \multirow{2}{*}{ Site muséal } & Événement et public & $\begin{array}{l}\text { Durée } \\
\text { (h.:min.:sec) }\end{array}$ \\
\hline \multirow{3}{*}{ MICR de Genève } & 21.06 .2019 - Visite guidée non-scolaire & $00: 47: 40$ \\
\cline { 2 - 3 } & 02.07 .2019 - Atelier webradio & $02: 55: 25$ \\
\cline { 2 - 3 } & 04.07 .2019 - Visite guidée scolaire & $01: 20: 25$ \\
\cline { 2 - 3 } & 04.07 .2019 - Atelier webradio & $02: 52: 08$ \\
\hline \multirow{2}{*}{$\begin{array}{l}\text { Mémorial national de la prison } \\
\text { Montluc de Lyon }\end{array}$} & $\begin{array}{l}19.09 .2019- \\
\text { Visite guidée séminaire étudiants ENS Lyon }\end{array}$ & $01: 52: 41$ \\
\hline \multirow{2}{*}{ Musée des Confluences de Lyon } & $\begin{array}{l}07.11 .2019 \\
\text { Visite libre séminaire étudiants ENS Lyon }\end{array}$ & $01: 32: 27$ \\
\hline $\begin{array}{l}\text { Musée des Confluences et } \\
\text { Mémorial national de la prison } \\
\text { Montluc (« duo ») }\end{array}$ & 07.01 .2020 - Visite guidée scolaire (2 gpes.) & $01: 31: 18 \times 2$ \\
\cline { 2 - 3 } & 07.01 .2020 - Visite guidée scolaire (2 gpes.) & $01: 26: 37 \times 2$ \\
\hline Musée des Confluences de Lyon & 03.03 .2020 - Atelier webradio & $01: 51: 53$ \\
\hline Total & 8 visites guidées et 3 ateliers webradio & $19: 08: 29$ \\
\hline
\end{tabular}

\subsection{La réalisation d'enregistrements de visites guidées}

Pour avoir un matériau qui favorise une enquête linguistique et sémiotique fine de la transformation de l'expérience et pour avoir des données qui garantissent le principe de réfutabilité de nos analyses, nous nous sommes appuyés sur un dispositif d'enregistrement audiovisuel développé par le laboratoire ICAR (UMR 5191) (Traverso, 2012 ; Mondada, 2014). Habituellement, en situation de visite guidée, plusieurs caméras sont mobilisées par 
des chercheurs de manière à avoir d'un côté une vue focalisée sur les guides et de l'autre un plan large sur l'espace interactionnel ${ }^{8}$.

Dans le cadre de notre projet, nous avons ajusté le dispositif avec l'utilisation d'une caméra opérée par les participants, en continuité avec les hypothèses de recherche présentées plus haut $(\S 1)$ et en vue d'une complémentarité d'un point de vue des chercheurs et d'un point de vue des visiteurs. Avec les termes du linguiste et anthropologue Kenneth Pike (1967), cette complémentarité assurée par un point de vue étique (chercheurs) et un point de vue émique (visiteurs) se révèle être une stéréoscopie dans l'espace interactionnel, sur laquelle nous reviendrons plus loin (§ 3.2). La consigne donnée s'est voulue le plus large possible, de manière à laisser aux visiteurs une marge de manœuvre dans l'utilisation de leur caméra : ils pouvaient l'opérer comme ils le voulaient, le temps qu'ils le voulaient avec la possibilité de l'éteindre et de nous la rendre ${ }^{9}$. Aussi, du fait d'une promiscuité dans le parcours, une complémentarité de deux caméras opérées par des chercheurs nous a semblé bienvenue, notamment pour pouvoir visualiser a posteriori les gestes et mimiques faciales. Nous avions donc deux caméras opérées par des chercheurs et une troisième caméra confiée aux participants en vue d'élaborer une vue multiscope des enregistrements. Ci-dessous, une capture d'écran représente le dispositif mobilisé lors d'une visite avec une classe de $5^{\text {ème }}$ au MICR de Genève ( $9^{\text {ème }}$ en Suisse).

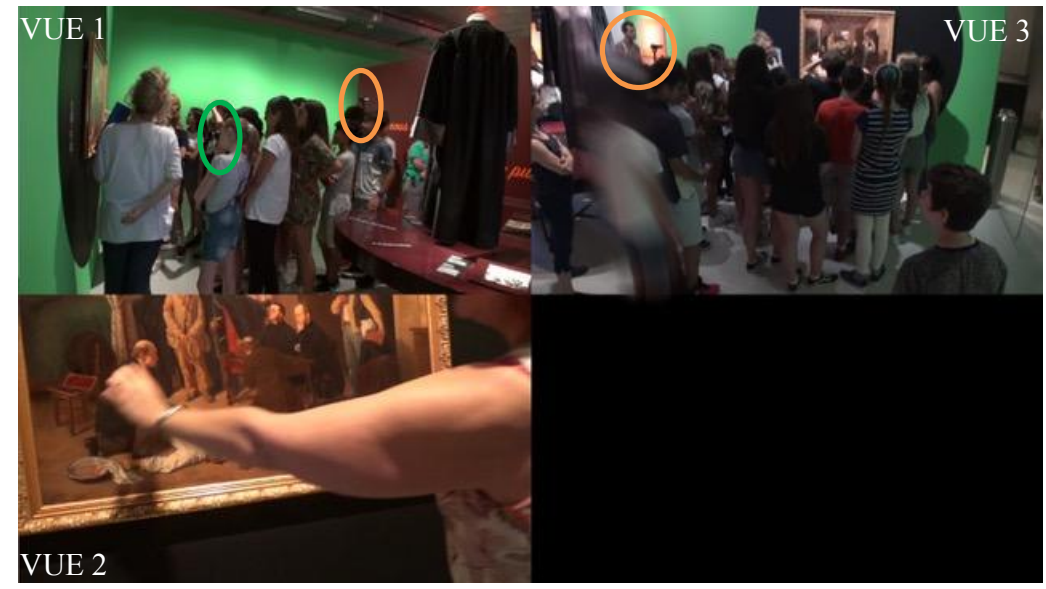

Fig. 2. Dispositif d'enregistrement - Visite guidée exposition Prison au MICR de Genève, le 04.07.19

Les vues 1 et 3 sont celles des caméras opérées par les chercheurs (encerclées en orange). Celles-ci ont été fixées à un monopied, non seulement pour garantir la stabilité de la prise de vue, mais aussi pour réaliser des plans en plongée afin de pallier les éventuels problèmes de profondeur de champ dus à la proximité des participants. La vue 2 est celle de la caméra confiée au groupe (encerclée en vert). Quant à elle, cette caméra était tenue " au poing ", de manière à en faciliter la prise en main par les participants et à réduire le caractère encombrant du dispositif. Ci-dessous, une capture d'écran permet de visualiser le même dispositif mobilisé au Mémorial national de la prison Montluc de Lyon, lors d'une visite avec des étudiants en master de sociologie et anthropologie. 


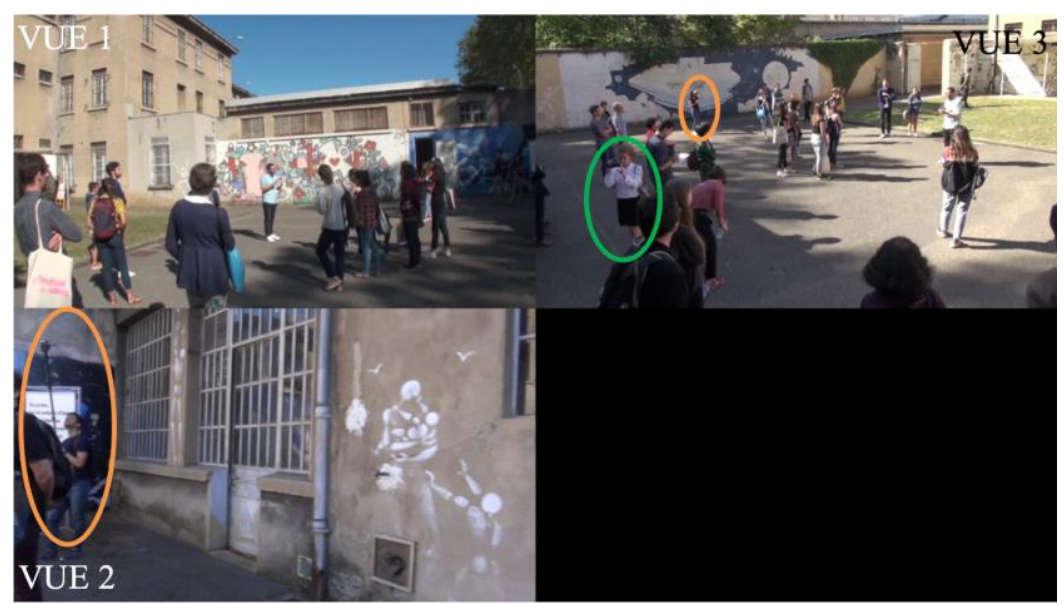

Fig. 3. Dispositif d'enregistrement - Visite guidée Mémorial national de la prison Montluc, le 19.09.19

\subsection{La réalisation d'enregistrements d'ateliers « Reporter au musée »}

Pour documenter les ateliers webradio « Reporter au musée » qui se sont déroulés au MICR de Genève, nous avons utilisé un dispositif que l'on pourrait caractériser d' " allégé ».

Ces ateliers d'environ 3 heures se composent, dans l'ordre :

- d'une introduction d'environ 30 minutes dans une salle de travail, où expliciter le déroulement de l'atelier et présenter les cinq chroniques webradio qui s'intitulent «agenda culturel» (les activités de la ville de Genève concomitantes à l'exposition Prison), «focus » (regard focalisé sur une partie de l'exposition, à travers une description aux auditeurs), " histoire d'objet » (description à des auditeurs d'un objet présent dans l'exposition, sa forme, son utilisation et ce qu'il raconte), «microtrottoir » (interview de visiteurs sur leur expérience de visite et restitution de leurs propos par des commentaires), « chronique scénographique » (restitution de la mise en scène de l'exposition qui cherche à mettre les visiteurs dans une certaine ambiance) ;

- d'une visite guidée d'environ 45 minutes conduite par un médiateur, pour faire se plonger les participants dans le parcours et dans le contenu de l'exposition ;

- d'une investigation et d'une mise en discours du matériau collecté d'environ 1 heure, en vue de préparer les cinq chroniques webradio ;

- d'une séquence d'enregistrement de webradio d'environ 30 minutes animée par les médiateurs et médiatrices du Musée des Confluences.

Afin de documenter ces ateliers, un dispositif de recueil un peu différent a donc été produit. Dans la salle de travail, deux caméras ont été placées sur trépied pour filmer les interactions sous deux angles différents. Ensuite, pour les phases prenant place dans l'espace d'exposition, les deux chercheurs-cadreurs ont d'abord suivi le groupe pendant la visite synthétique (fig. 3) puis ont chacun suivi un binôme d'enfants lors de la phase d'investigation. Nous avons fait le choix de ne pas donner de caméra "visiteur», les enfants ayant déjà des carnets pour prendre des notes pour leurs chroniques. Par contre, les enfants des binômes suivis étaient équipés de micro-cravate afin de pouvoir entendre leurs propos a posteriori : les caméras opérées par les chercheurs étant parfois relativement éloignées des binômes, le son capté ne permet pas une écoute claire de leur parole. 


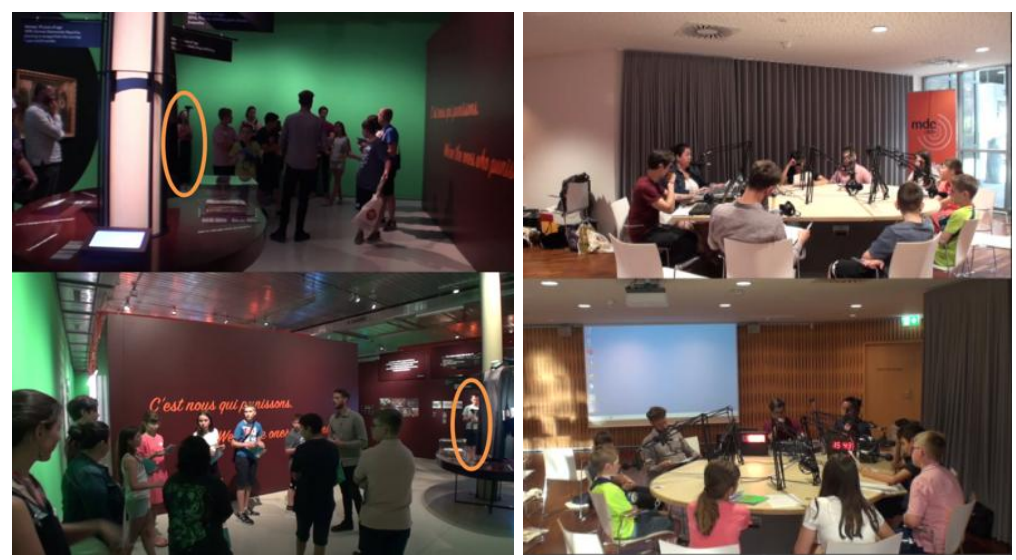

Fig. 4. Dispositif d'enregistrement - Ateliers webradio « Reporter au musée » - MICR de Genève, le 02.07.19

En continuant de retracer ici notre pratique de constitution et de traitement des données, une réflexion s'impose sur les choix opérés quant à l'ajustement du dispositif entre sa conception en amont des captations et sa mobilisation dans le cours même des enregistrements.

\section{Sites à parcourir, documents à produire et extraits à réancrer}

Nous proposons ainsi de mettre en perspective (3.1) les tensions à l'œuvre dans le parcours des différents sites entre la phase de préparation et de repérage des lieux et la pratique des enregistrements in vivo, (3.2) les questions qui se sont imposées à nous dans le posttraitement des enregistrements et (3.3) la consolidation des différents niveaux d'analyse, entre un regard sur le déploiement des visites dans leur ensemble et la réalisation locale de courts extraits en vue d'une analyse à un niveau de granularité fin.

\subsection{Parcourir le site muséal : du repérage des lieux à la visite in vivo}

Nous avons pu apprécier l'évolution de nos rapports aux trois sites muséaux dans la constitution et la mobilisation du dispositif, entre un lieu dont les usages sont programmés selon des scénarios institutionnellement cadrés et un espace dont les caractéristiques propres émergent de la concaténation des expériences locales que nous nous en faisions. Aussi, un repérage des lieux s'avérait nécessaire dans la négociation de notre propre présence et de notre pratique vis-à-vis de celle des autres agents avec qui nous collaborons, sur plusieurs plans. Sur le plan pratique, il s'agit d'anticiper les éventuels écarts entre la projection que nous nous faisons du terrain et la matérialité effective des différents sites. Sur le plan épistémique, il nous paraît nécessaire d'avoir une connaissance du contenu des visites non seulement pour comprendre le rythme de la progression thématiques et nous projeter ainsi le tempo de notre pratique future, mais aussi pour une curiosité intellectuelle vis-à-vis des discours qui s'y déploient. Sur le plan socio-symbolique, le repérage tient pour nous une place primordiale en ce qu'il permet un échange préalable quant aux métiers et aux sensibilités des différentes personnes que nous sollicitons et, par là même, participe d'un respect des pratiques des institutions et des individus impliqués dans notre recherche. Et, si l'on ne peut pas se permettre d'arriver à l'improviste, nous avons dû élaborer une forme d'improvisation inhérente à la cogestion d'un cours d'action in vivo, en faisant face aux continuités et aux discontinuités entre la trame d'actions programmée suite aux 
repérages et la manière dont la constitution des enregistrements se déroule de manière effective.

Nous identifions ainsi plusieurs plans vis-à-vis desquels s'est manifesté cet imprévu, aussi bien pour nous-mêmes que pour le groupe dans son entier. Sur le plan technique, au MICR de Genève, nous avons dû faire face aux limites des potentialités des micros HF, en ce que la salle d'exposition se trouve au sous-sol du bâtiment dont la structure est réalisée dans un béton épais. Dans l'enregistrement des ateliers, lorsque nous suivons des binômes à distance l'un de l'autre, il arrive que l'émetteur du micro HF se trouve dans la salle d'exposition au sous-sol alors que le récepteur est resté à l'étage, au rez-de-chaussée : le signal ne passe pas et les échanges que nous avions l'intention d'enregistrer avec ces micros ne sont accessibles qu'à travers le micro de la caméra. Sur le plan du déroulement de l'activité de visite guidée, nous devons nous adapter aux contingences du terrain en prenant en compte les contraintes qui s'imposent aux participants de manière endogène (des contraintes internes à l'activité, telle que la présence d'autres personnes qui rend impossible notre positionnement à un certain endroit de l'espace et qui nous empêche d'opérer une certaine prise de vue) ou exogène (des contraintes externes à l'activité, telle que la place de cette visite dans l'organisation d'une journée dans son ensemble). De manière non anecdotique, il est notamment arrivé qu'un malentendu acoustique provoque un malentendu organisationnel, lorsque deux guides avaient prévu de finir à quinze heure et quart la visite qu'elles menaient toutes les deux en parallèle, avec deux sous-groupes, et se sont retrouvées délaissées par les visiteurs qui s'étaient donnés rendez-vous à quinze heures au car. Sur le plan socio-affectif et socio-symbolique de la captation audio et vidéo de l'interaction, il n'est pas rare que des membres d'un groupe ne souhaitent pas être filmés. Nous devons alors adapter notre cadrage de la scène en faisant en sorte de les situer hors du champ de la caméra. Au contraire, dans une version plutôt euphorique, d'autres personnes s'approprient la caméra que nous leur avons confiée en négociant notre pratique d'observation et notre modalité de participation en jouant, en quelque sorte, au « filmeurfilmé », en opérant des zooms sur les chercheurs en train de réaliser les enregistrements de l'interaction.

Il faut alors noter que de telles dynamiques n'ont été observées qu'au cours des visites de l'exposition Prison qui ont eu lieu au MICR de Genève. Sans avoir pour autant de données consistantes permettant de vérifier cette hypothèse, nous nous demandons tout de même si cette hétérogénéité de l'éthique des participants ne serait pas un indice d'une différence de l'expérience phénoménologique et affective de ces sites muséaux. Autrement dit, à partir des autres enregistrements que nous constituerons, nous pourrons étudier si l'absence d'un jeu entre les participants à travers cet objet pourrait être " conditionnée », d'une certaine manière, par l'identité spatiale et historique, sociale et symbolique, de ce lieu de mémoire qu'est le Mémorial. Notre étude des représentations sur les prisons en contexte muséal se déploie donc dans un parcours complexe des trois sites où articuler une perspective sur l'évolution des pratiques d'un même site à travers la mise en regard les différentes pratiques qui s'y réalisent avec les pratiques des autres sites, à une perspective transversale aux pratiques des différents sites.

\subsection{La vue multiscope : synchroniser et mettre en relief les points de vue}

Ce parcours complexe est à aborder sur le plan praxéologique et discursif, où articuler les points de vue des participants et des chercheurs dans l'espace interactionnel, aussi bien in vivo, comme nous venons de le dire, qu'en post-traitement. Ce post-traitement des diverses sources d'enregistrement audiovisuelles a été réalisé selon une méthodologie largement éprouvée au laboratoire ICAR (Jouin-Chardon et al., 2010 ; Thiburce, 2018), notamment grâce au travail de la Cellule Corpus Complexes ${ }^{10}$. Pour pouvoir rendre compte des différents points de vue réalisés d'une même situation, nous synchronisons toutes les 
sources audio et vidéo entre elles pour qu'elles aient chacune le même timing de début et de fin, tout en les exportant comme des fichiers distincts. Nous obtenons alors une vue 1, une vue 2 , une vue "visiteur» et plusieurs pistes audio, chacune se référant au même événement. C'est dans un deuxième temps que nous choisissons de faire le montage multiscope de ces pistes afin d'en permettre une lecture synchrone. La disposition de ces différentes vues, le choix de mixage des différentes pistes audio permet au chercheur de repositionner les différents points de vue instaurés dans le cours de l'interaction pour enquêter a posteriori sur l'expérience de visite, à partir des indices linguistiques et sémiotiques documentés. Réalisée entre synchronisation sans montage et articulation stéréoscopique, cette vue multiscope cherche à élaborer une mise en commun des vues prises in situ non pas comme une parataxe des observations, mais comme une mise en syntaxe des points de vue en coprésence. La possibilité de retracer l'ajustement progressif et séquentiel des pratiques des individus les uns par rapport aux autres est une ressource fondamentale pour étudier les liens de réciprocités en jeu dans les dynamiques de solidarisation et de désolidarisation dans la socialisation d'un point de vue dans le cours d'une visite guidée en groupe. Cette vue multiscope favorise ainsi une mise en relief des perspectives individuelles dans et sur la scène de l'interaction. La dynamique de cette mise en relief n'est pas du même ordre que celle d'une reconstitution d'une scène pratique à partir de l'interprétation d'un pictogramme (Fontanille, $2008: 77)^{11}$, où l'on chercherait à rendre la densité d'une interaction à partir d'une mise à plat qui aurait été effectuée en amont. Ici, cette mise en relief est préalable à toute mise à plat que l'on pourrait éventuellement opérer à travers une schématisation de la scène interactionnelle.

Un tel phénomène peut être illustré concrètement à partir des enregistrements constitués de la visite scolaire au MICR de Genève (fig. 1). Un peu plus loin dans cette visite, il est en effet intéressant d'observer comment une complémentarité des points de vue sur des objets $\mathrm{du}$ quotidien des personnes incarcérées se déploient dans l'espace interactionnel. Plus particulièrement, il s'agit d'observer à travers la vue multiscope les tensions entre le guidage de l'attention réalisé par la médiatrice à travers la multimodalité de la parole-eninteraction et la focalisation attentionnelle opérée par les visiteurs sur les objets thématisés en discours. Pour cet extrait, nous proposons de pister et de retracer la gestion de cette mise en relation de différents objets à travers une transcription du discours verbal et des captures d'écran permettant un accès à la dimension praxéologique de l'interaction. Selon les conventions mobilisées ${ }^{12}$, GUI réfère aux prises de parole de la guide et VE à celles des élèves. Dans la transcription, le symbole \#1 positionne la capture d'écran 1 dans le cours de l'interaction.

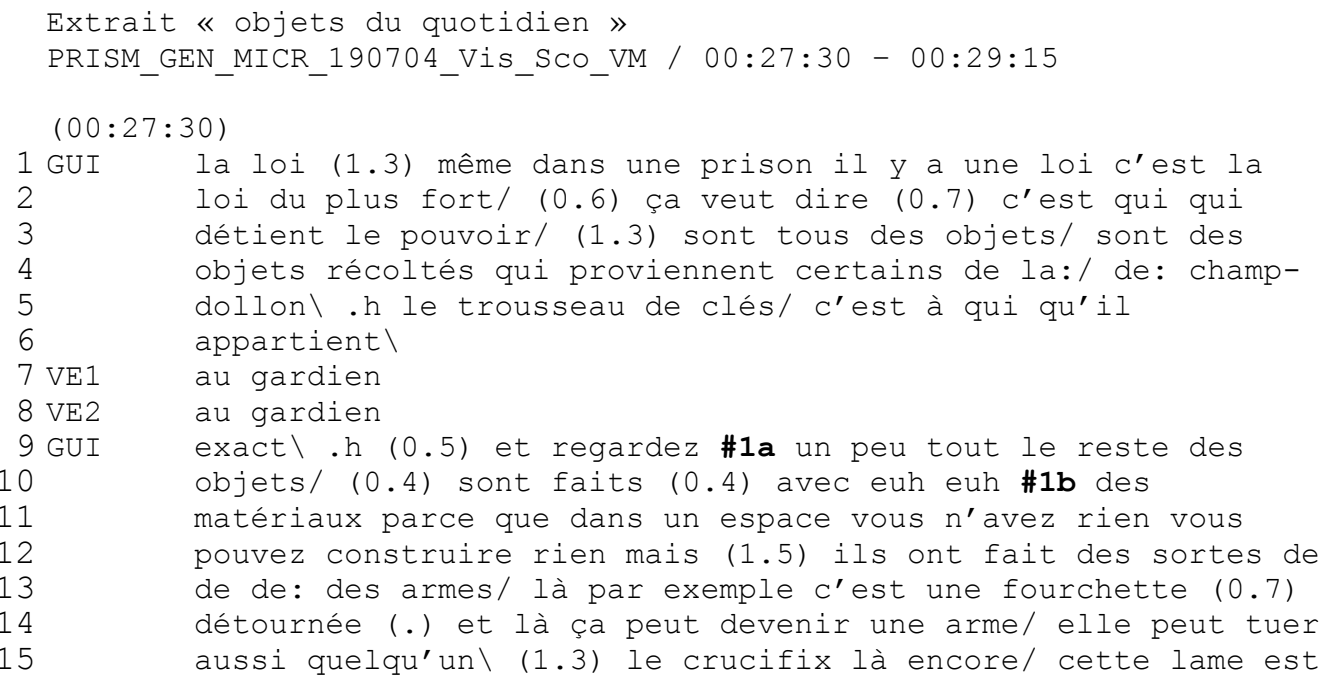




\begin{abstract}
cachée (.) dans le crucifix là c'est une chaussure mais si vous regardez bien là c'est une lame aussil (.) cachée (0.7) voilà tous des objets/(0.5) utilisés dans la du quotidien (.) là/ c'est une frondel et là je sais pas si vous la reconnaissez c'est une semelle de: adidas/

Pour ce passage, il est intéressant d'observer les articulations entre, d'une part, le discours de la médiatrice qui opère un regroupement entre des objets selon leur propriété formelle et leur finalité pratique et, d'autre part, la mise en syntaxe visuelle de ces objets opérée par la caméra confiée au groupe. Des lignes 1 à 24, alors que la guide met en relation le trousseau de clé disposé au centre avec une première fourchette, un crucifix, une chaussure puis une fronde, symboles des rapports de force et de pouvoir entre les surveillants pénitentiaires et les détenus, la personne qui tient la caméra du groupe réalise un plan large sur la table où sont exposés ces objets détournés en armes (\#1a et \#1b, cadre orange). En \#1a, on voit la guide pointer un objet de sa main droite (flèche orange) tout en en regardant un autre (flèche verte), tandis qu'en $\# 1$ b, regard et pointage de l'auriculaire sont en continuité.

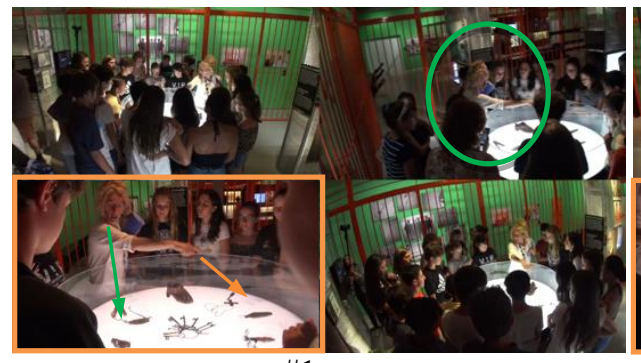

\#1a

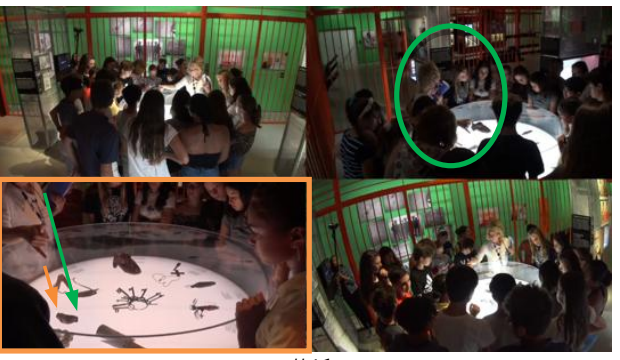

\#1b

Ce n'est que lorsque la guide thématise la présence de poings américains (1. 24) qu'un recadrage et un zoom avant sont opérés sur l'un des objets de la table (\#2a, \#2b, \#2c et \#2d).

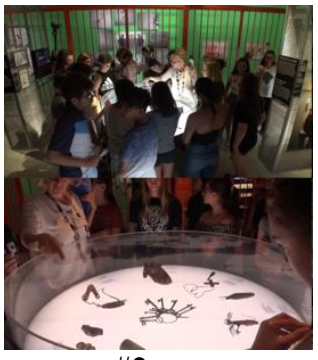

\#2a

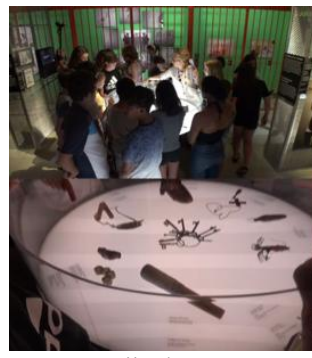

\# 2b

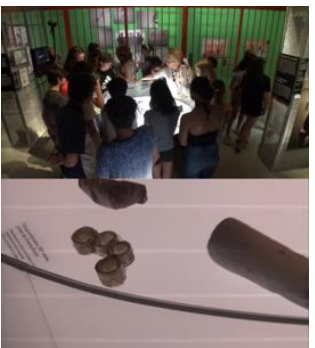

\#2c

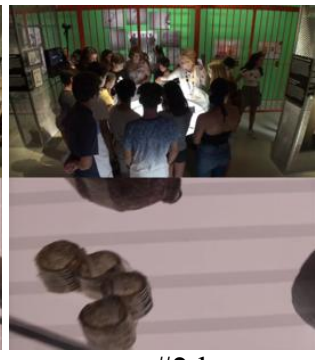

\#2d 
On observe ici que la guide n'est plus dans le champ visuel de l'élève, son discours passe au deuxième plan, et les objets deviennent le centre de l'attention dans une forme d'exploration et d'appropriation individuelle. Et quand la guide propose de s'orienter vers un autre type de détournement de fourchettes en grappin (1.27), l'élève qui opère la caméra continue de cadrer sur les poings américains (\#3a), opère un léger zoom arrière (\#3b) puis cadre vers le grappin en question (\# $3 \mathrm{c}$ et \#3d).
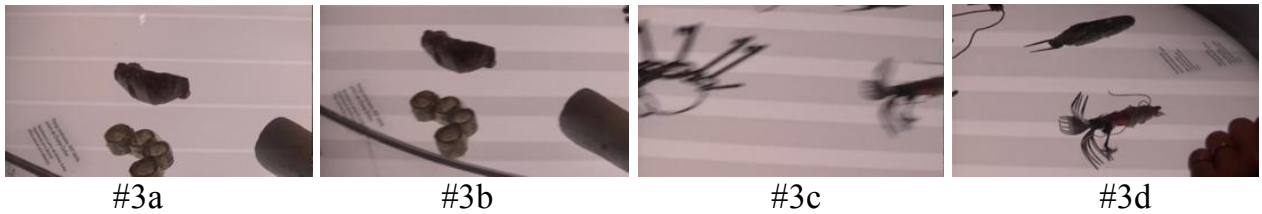

Lorsque la médiatrice termine sa focalisation sur ce grappin et explicite aux élèves qu'ils auront du temps plus tard pour se faire leur propre parcours, l'élève opère un zoom arrière (\#4) et un recadrage de la prise de vue sur les actions de la guide dans le groupe (\#5).

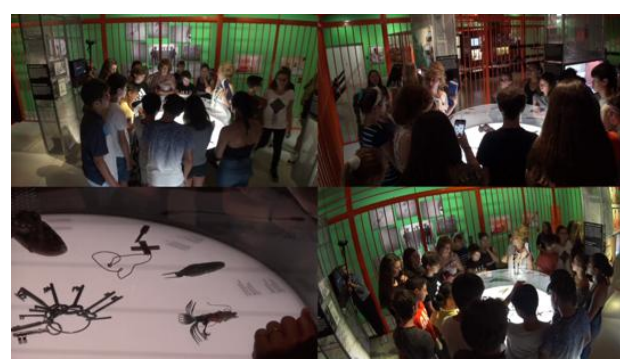

\#4

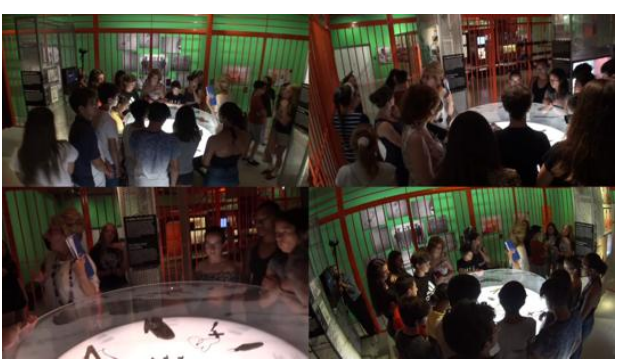

\#5

Fig. 4. Captures d'écran de l'extrait «objets du quotidien » - Visite guidée exposition Prison au MICR de Genève, le 04.07.19, avec une classe de collège

Ainsi, cette vue multiscope qui articule le point de vue sur les participants opéré par les chercheurs et le point de vue de l'intérieur du groupe déployé par la caméra confiée aux participants, montre sa pertinence sur au moins deux plans. D'une part, sur le plan logistique et pratique, la vue multiscope permet de situer un accès rapproché aux objets perçus, commentés et thématisés en discours dans l'espace de l'interaction. D'autre part, sur le plan sémiotique et praxéologique, la vue multiscope met en visibilité les porosités entre ce que dit la guide sur les objets en question et les modalités actionnelles par lesquelles les coparticipants gèrent leur perception de l'environnement par leur corps (perception incarnée) et par l'utilisation de la caméra (perception instrumentée). Si nous ne disposions que notre point de vue, nous n'aurions que peu d'accès aux focalisations attentionnelles opérées par les participants (ce sur quoi porte leur regard, gestion de la profondeur de champ, mise en syntaxe de différents éléments coprésents). Et si nous ne disposions que du point de vue des participants, il serait plus difficile de situer les actions de chacun dans l'environnement de l'interaction.

Dans une attention portée à l'affinement de notre épistémologie, il sera opportun de mettre en regard notre dispositif de constitution de données avec d'autres dispositifs mobilisés en analyse des interactions (Dufiet, 2012), dans la suite de notre recherche. Aussi, le caractère multiple et hétérogène des points de vue instaurés au cours des interactions ne saurait être uniquement érigé en principe de base et reste à étudier à partir des traces que l'on en a. Cela demande alors de décrire les différentes modalités d'usages des caméras et micros à partir d'une étude locale des extraits choisis et d'une perspective transversale aux visites documentées. Cette articulation entre l'extraction d'un passage et la 
vue sur l'ensemble d'une visite est un aspect crucial dans la méthodologie d'analyse que nous cherchons à développer dans le cadre de notre projet.

\subsection{Ancrer et annoter avec ELAN : usages tacites et réappropriation du logiciel}

Les enregistrements audiovisuels des visites ont pour objectif de nous fournir un corpus d'attestation d'interactions à étudier ${ }^{13}$. Pour mener à bien cette étude, nous produisons des descriptions et des analyses des interactions à partir d'extraits isolés ou bien d'extraits regroupés en collection (Mondada, 2008) ${ }^{14}$.

En vue d'opérer une extraction contrôlée et raisonnée, nous avons eu recours à la constitution de synopsis de chaque visite avec le logiciel Microsoft Excel ${ }^{\circledR}$. Ces synopsis entre paradigmatique et syntagmatique des visites favorisent (i) une vision sur le traitement d'une section du parcours d'exposition spécifique à une visite ou partagé avec d'autres, (ii) et une relocalisation d'un passage dans l'ensemble du corpus. Entre la caractérisation locale d'une situation spécifique et la perspective panoptique sur les différentes situations enquêtées, nous avons identifié trois axes qu'il pourrait être pertinent d'étudier de manière transversale à chacun des enregistrements :

- thèmes, topoï et isotopies : dans une perspective énonciative (descriptive, narrative, argumentative), quels sont les sujets traités en discours par l'exposition, par la médiation des guides et par les prises de paroles de visiteurs qui reviennent dans le cours d'une même visite? Et dans plusieurs visites?

- stratégies énonciatives : par quelles ressources linguistiques et sémiotiques les différents acteurs de la scène énonciative (institutions muséales, médiateurs, visiteurs) gèrent-ils leurs points de vue respectifs en instaurant un référentiel ou bien partagé (collectif ayant une expérience d'un monde en commun) ou bien propre à chacun (positionnement énonciatif à travers l'assomption d'un supposément singulier) ? comment les médiateurs gèrent-ils leur adresse à un groupe de personnes particulier en énoncé?

- mobilisation des ressources spatiales : une dialectique s'instaure dans la production d'un discours à destination des publics où, d'un côté, les médiateurs décrivent les objets exposés et les lieux d'exposition afin d'en dire quelque chose, d'en prédiquer des valeurs et d'en guider l'interprétation ; d'un autre côté, les médiateurs s'appuient sur l'espace où ils se trouvent et sur les objets qui y sont présentés en leur cédant la parole, en les laissant parler de eux-mêmes et en laissant les visiteurs s'en faire une exploration personnelle.

Ce format de synopsis réalisé avec le logiciel Microsoft Excel ${ }^{\circledR}$ permet de retracer la trame thématique de chaque visite de manière chronologique scandée par des « séquences de médiations » et des « séquences de déplacement» (Ravazollo, 2012). En revanche, il affiche quelques limites qui nous ont fait le remplacer par un autre logiciel favorisant une autre modalité d'annotation. D'une part, il ne permet pas de procéder à l'annotation fine du corpus en vue de procéder a posteriori à des requêtes systématisées de l'ensemble des enregistrements. D'autre part, les balises temporelles marquées sur Excel pour identifier le début et la fin d'une séquence ne sont pas directement alignées aux fichiers sources audiovisuelles, ce qui exige un parcours des deux documents en parallèle qui s'avère lourd et peu ergonomique. Nous avons alors choisi de coupler la phase de transcription des échanges à la réalisation des synopsis à travers l'annotation des enregistrements sur le logiciel ELAN ${ }^{15}$, chacune alignée au signal audiovisuel, de manière à pouvoir les situer chronologiquement au sein d'une même fenêtre. À partir de la transcription et de l'annotation du corpus avec ce logiciel spécifique, on cherche à coupler une caractérisation des dynamiques linguistiques et sémiotiques (en transcrivant les paroles et les gestes des 
participants, leur rapport aux objets dans l'environnement de l'interaction) à celle des pratiques muséales (en mettant en visibilité des dynamiques propres à l'activité de médiation dans les différents musées).

Ce logiciel s'avère aussi tout à fait propice à l'emboîtement et la hiérarchisation des lignes d'annotations (dénommées «acteur»). Les lignes nous permettent de définir la production linguistique et gestuelle de chaque participant à l'interaction et, comme le nombre de participants varie d'une visite à l'autre, on peut ajouter ou supprimer des lignes correspondant à leur production respective. Aussi, en définissant les lignes nécessaires, transversales à toutes les interactions documentées (telles que les séquences de médiation et des séquences de déplacement ou encore la thématisation linguistique et praxéologique de la caméra), nous pouvons opérer des requêtes sur la totalité du corpus à travers un ensemble de termes qui caractérisent ces séquences et ces actions. Les lignes peuvent être indépendantes les unes des autres, ou entretenir une relation de dépendance. Les relations de dépendance entre les acteurs peuvent être de quatre types et sont définies au moyen de «types de pistes». Dans notre situation d'interaction et de visite au musée, nous définissons certaines dépendances entre acteurs qui sont communes et transversales à l'ensemble des données. Il s'agit là de notre grille d'annotation ELAN composée de 13 «acteurs» d'annotations auxquels s'ajoutent les lignes acteurs des locuteurs pour la transcription. Nous utilisons ce qu'ELAN appelle du "vocabulaire contrôlé » non seulement pour éviter les erreurs de saisie et mais surtout pour uniformiser les codages lorsque les annotations se répètent. Illustrons ci-dessous cet emboîtement.

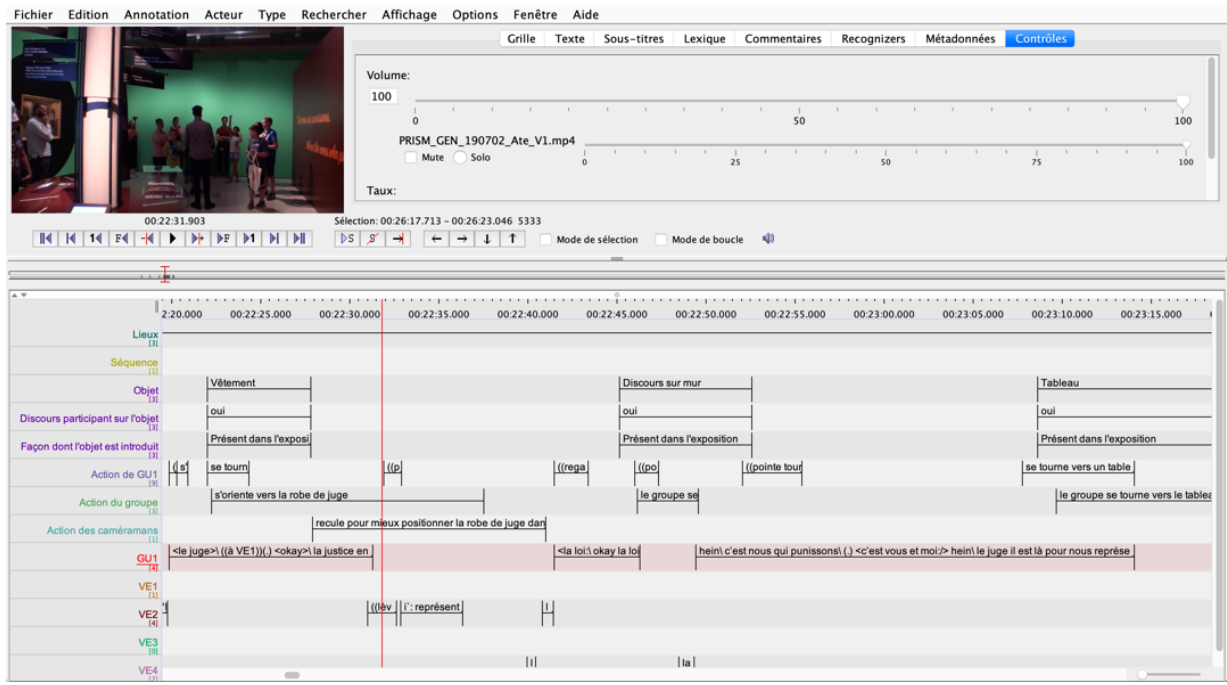

Fig. 5. Dépendance et indépendance des lignes d'annotation sur le logiciel ELAN

Ce vocabulaire contrôlé est présent pour les lignes «Lieux », "Séquences » et «Objet», dernière ligne de laquelle dépendent les lignes «Discours participant sur l'objet » et « Façon dont l'objet est introduit ».

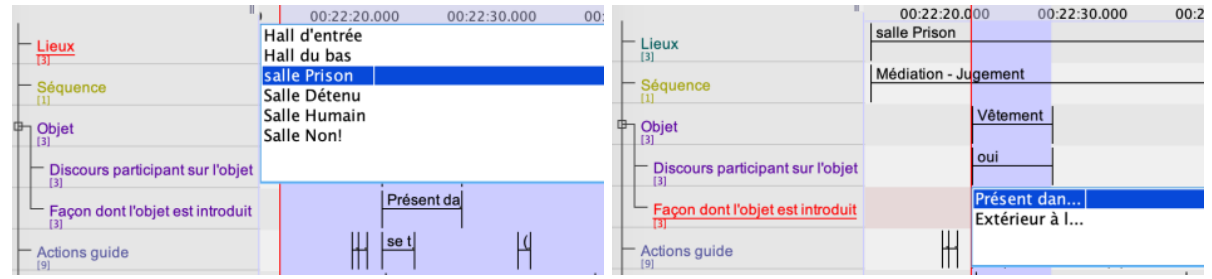

Fig. 6. Dépendance et indépendance des lignes d'annotation sur le logiciel ELAN - Vue détaillée 
Pour chacune de ces lignes, nous identifions un choix réduit de propositions d'annotation permettant de définir le lieu de l'exposition (hall d'entrée du musée, hall du bas du musée, différentes salles de l'exposition) où se déroule la séquence à laquelle on s'intéresse (questions logistiques, introduction à la visite, séquence de médiation, de déplacement), ainsi que les artefacts qui font l'objet du discours et de l'attention des participants (en précisant si ces objets sont coprésents dans l'environnement ou bien s'ils sont rendus présents seulement en discours). Une telle utilisation du logiciel ELAN pour les trois sites muséaux où nous enquêtons demande alors de penser les invariants de la pratique de médiation en espace muséal (quelles sont les lignes d'annotations nécessaires pour étudier les pratiques des différents musées dans leur transversalité ?) ainsi que leurs caractéristiques respectives (sachant que les expositions diffèrent d'un musée à l'autre, aussi bien sur le plan architectural qu'au niveau des types d'objets mobilisés dans les deux expositions qui nous intéressent).

Par exemple, si l'on s'intéresse aux transformations d'une visite à l'autre, il peut être pertinent d'opérer une requête sur au moins deux visites qui couplerait les plans quantitatif (recherche du nombre d'occurrences d'un terme) et qualitatif (recherche des termes cooccurrents à travers un empan plus ou moins large). Dans l'état actuel de l'avancement du projet, le nombre de transcription réalisées et le niveau de granularité de ces transcriptions ne nous permettent pas de proposer une analyse détaillée dans le présent article. Une étude ultérieure sur le corpus dans son ensemble permettra donc de démontrer la force heuristique du recours à ELAN pour nos questions de recherche et de tester la pertinence de cette extension de son utilisation, moins conventionnelle que la réalisation des transcriptions et des requêtes qui relèvent quant à eux d'usages tacites de ce logiciel.

\section{Ouvertures / Diversifier les méthodes pour les consolider}

Dans l'évolution des pratiques de la recherche, cette utilisation du logiciel ELAN participe selon nous d'une exploration active de nouvelles potentialités de l'outil d'annotation de données audiovisuelles, exploration elle-même liée à la formulation des questions de recherche et à l'émergence des pistes d'enquête au fil de l'étude. Cette extension des usages, ordinaire dans notre appropriation des objets du monde, interroge en effet l'établissement d'un lien réciproque entre un travail théorique sur les pratiques langagières à travers la généralisation d'observations dans de multiples contexte et une étude locale et outillée des représentations des prisons sur les trois sites muséaux où se déploie notre recherche. Une telle réflexion de notre part sur la transformation des rapports entretenus aux outils de la recherche ne tente en aucun cas de s'imposer comme un précepte devant être adopté par tous; elle vise plutôt à rendre compte d'un principe régulateur dont nous faisons l'expérience au quotidien, dans la mise au travail de nos approches théoriques et pratiques de la recherche en équipe. De la même manière que la découverte des potentialités d'un objet tel que la caméra est intimement liée aux situations auxquelles on se trouve confronté lors de la constitution des enregistrements, en amont de l'analyse, la pertinence des outils permettant d'annoter et de décrire des enregistrements audiovisuels d'interactions d'activités spécifique se négocie au fil de leur mobilisation au fil des recherches, en aval des données.

Pour le contexte muséal qui nous intéresse, entre le fait de travailler uniquement à partir d'un texte déjà constitué comme le catalogue d'exposition et le fait de s'immerger dans les relations sociales en situation de visites guidée, c'est la recherche en sciences du langage en tant que pratique collective qui se trouve être problématisée. La diversité des voix et des points de vue disciplinaires qui s'exprime dans notre recherche, au sein d'un paysage plus vaste, vise à répondre à la multiplicité des acteurs et à l'hétérogénéité des situations auxquels nous avons été confrontés. Aussi, au regard de la complexité langagière qui est à l'œuvre dans la mise en discours des pratiques carcérales au musée, notre enquête ne 
saurait se passer de la pluralité des méthodes présentes au sein des sciences du langage et, plus largement, en sciences sociales. Mettre à l'épreuve cette pluralité dans une approche située des expériences des publics participe moins d'une mise en concurrence des outils de la recherche que d'une consolidation de pratiques au sein d'un domaine scientifique.

En sollicitant les institutions muséales pour enquêter sur les pratiques culturelles ou en travaillant avec elles dans leurs missions d'évaluation et d'étude de la réception des publics, une problématisation autoréflexive de notre pratique de terrain se trouve nécessairement à l'œuvre au fil de l'étude. De la constitution des objectifs à la formulation d'un bilan ou d'un rapport, en passant par l'analyse fine des expériences de visites, la mise en œuvre d'une approche transversale aux sciences du langage se veut être une consolidation de manières de faire la recherche, tout en transmettant des outils aux étudiants dans les cursus universitaires à travers une sensibilisation à de nouvelles situations d'apprentissage, en continuité avec l'évolution des pratiques culturelles environnantes. Dans la volonté de cultiver les relations avec les acteurs du terrain, on tente également de consolider les liens entre les pratiques de la recherche en sciences sociales (en testant le caractère partagé des méthodes entre disciplines et du caractère extensif des outils sur divers terrains) et les pratiques de recherche et développement au sein des institutions culturelles muséales (en participant notamment à l'analyse des pratiques de médiation auprès des publics). Ce projet constitue pour nous un banc d'essai pour le développement d'une méthode transversale à la sémiotique des pratiques et à l'analyse des interactions qui serait à même de proposer une approche construisant des liens entre programmation de l'action (constitution d'un itinéraire adapté à un public) et ajustement en situation (prise en compte des compétences interactionnelles des participants).

\section{Références bibliographiques}

Basso Fossali, P. (2008). Vissuti di significazione. Temi per una semiotica viva. Pisa: ETS.

De Stefani E. (2010). Reference as an interactively and multimodally accomplished practice: organizing spatial reorientation in guided tours. In Pettorino M. et al. (ed.), Spoken communication between symbolic and deixis. Newcastle: Cambridge Scholars Publishing, pp.137170.

Dufiet J.-P. (éd.) (2012). Les visites guidées. Discours, interaction, multimodalité. Trento: Labirinti.

Fontanille, J. (2008). Pratiques sémiotiques. Paris : PUF.

Garfinkel, H. (1967). Studies on ethnomethodology. Englewood Cliffs (NJ): Prentice-Hall.

Heritage, J. C. (1984). Garfinkel and Ethnomethodology. New York (NJ): Polity Press.

Jouin-Chardon, E. et al. (2010). Contraintes technologiques sur les enregistrements de corpus et analyse des cadres de participation. Pratiques, ${ }^{\circ} 147-148$, pp. 53-81.

DOI : https://doi.org/10.4000/pratiques.1606

Jutant C. (2011). S'ajuster, interpréter et qualifier une pratique culturelle: Approche communicationnelle. Thèse de doctorat, sous la direction d'Y. Jeanneret et de B. Schiele, Université d'Avignon et des pays de Vaucluse, Université du Québec à Montréal, programme international de doctorat conjoint en muséologie, médiation et patrimoine.

Kreplak, Y. (2014). Entrer dans l'espace de l'œuvre : une approche praxéologique de l'accrochage. Dans Mondada L. (dir.), Corps en interaction : participation, spatialité, mobilité. Lyon : ENS Editions, pp. 357-395.

Lallier, C. (2009). Pour une anthropologie filmée des interactions sociales. Paris : Éditions des archives contemporaines.

Landowski, E. (2006). Les interactions risquées. Nouveaux Actes Sémiotiques, n¹01-103, Limoges : Pulim

Licoppe, C (2012). Les formes de la présence. Revue française des sciences de l'information et de la communication [En ligne], 1. URL : http://rfsic.revues.org/142 
Lynch, M. (2002). From naturally occurring data to naturally origanized ordinary activities: Comment on Speer. Discourse Studies, vol. 4, n 4, pp. 531-537.

Mondada, L. (2006). Video recording as the reflexive preservation and configuration of phenomenal features for analysis. In H. Knoblauch, B. Schnettler, J. Raab \& H. G. Soeffner (eds.), Video Analysis: Methodology and Methods. Qualitative Audiovisual Data Analysis in Sociology. Berne : Peter Lang, pp. 51-68.

Mondada, L. (2008). Contributions de la linguistique interactionnelle. In Durand J., Habert B., Laks B. (éds.), Discours, pragmatique et interaction. Paris : EDP Sciences, pp. 881-897.

Mondada, L. (2012). Organisation multimodale de la parole-en-interaction: pratiques incarnées d'introduction des référents. Langue française, 175 (3), pp. 129-147

Mondada, L. (2014). Corps en interaction : une approche séquentielle et multimodale du langage en interaction. Dans Mondada L. (dir.), Corps en interaction : participation, spatialité, mobilité. Lyon : ENS Editions, pp. 11-64.

Pike, K. (1967). Language in relation to a unified theory of the structure of human behavior. The Hague: Mouton.

Rabatel, A. (2017). Pour une lecture linguistique et critique des médias : Empathie, éthique, point(s) de vue. Limoges : Lambert-Lucas.

Ravazzolo, E. (2012). Les manifestations de l'interaction entre le guide et son public en situation de visite guidée. In J.-P. Dufiet (éd.), Les visites guidées. Discours, interaction, multimodalité. Trento: Labirinti, pp. 85-120.

Sacks, H., Schegloff, Emmanuel, A. \& Jefferson, G. (1974). A Simplest Systematics for the Organization of Turn-Taking for Conversation. Language, 50, 4, pp. 696-735.

Schegloff, E. A. (1987). Analyzing single episode of conversation: an exercise in conversation analysis. Social Psychology Quarterly, 50, pp. 101-114.

Schmitt, D. (2018). L'énaction, un cadre épistémologique fécond pour la recherche en SIC. Les Cahiers du numérique, vol. 14, $\mathrm{n}^{\circ}$ 2, pp. 93-112.

Thiburce, J. (2018). Camera fabula. La caméra, outil et objet sémiotique complexe dans l'interaction. In J. Thiburce et B. Ursi (éds.), SHS Web of Conferences, vol. 52, ICODOC 2017 : Les ressources mobilisées en interaction, EDP Sciences. DOI : https://doi.org/10.1051/shsconf/20185203003

Traverso, V. (2012). Délimitation et partage des espaces : usage des annonces dénominatives désignatives dans la visite guidée. In J.-P. Dufiet (éd), Les visites guidées. Discours, interaction, multimodalité. Trento: Labirinti, pp. 55-86.

Traverso, V. \& Ravazzolo, E. (2016). Définitions ostensives co-construites. Le cas de la visite guidée. Langages, 204 (4), pp. 43-66.

Varela F. ([1996] 2004). Quel savoir-faire pour l'éthique? Action, sagesse et cognition. Paris : La Découverte.

\footnotetext{
${ }^{1}$ Aussi, le terrain d'étude des expériences de visite telles qu'elles sont vécues et cogérées par les participants s'avère être tout à fait pertinent pour travailler sur les transformations des relations de confiances et de croyances entre les agents sociaux et les institutions garantes d'un discours artistique et scientifique ancré socialement et historiquement :

les enjeux autour de l'expérience de visite méritent qu'on s'y attarde car le musée présente plusieurs avantages qui en font un terrain d'étude singulier. Il y a tout d'abord la confiance exceptionnelle que les visiteurs accordent aux collections et à la parole du musée (cartels, textes, vidéos, nouveaux dispositifs de médiation...) qui confère au musée une propriété particulière : le musée dit des choses vraies sur des vraies choses.

(Schmitt, $2018: 94$ )

${ }^{2}$ Notre projet intitulé PrisM (Prisons et Musées) porte sur les représentations des prisons en contexte muséal, mené en partenariat avec le Musée de la Croix-Rouge et du Croissant-Rouge de Genève (Suisse) et le Musée des Confluences de Lyon (France) qui, avec le Deutsches Hygiene Museum de Dresde (Allemagne) coproduisent tous les trois l'exposition internationale et itinérante Prison. Dans ce cadre, le terme représentations est à lire tant dans une acception sémiotique (les représentations sont les médiations et les ressources matérielles mobilisées dans la constitution d'un parcours d'exposition) que dans une acception axiologique (dans la négociation des valorisations entre les institutions muséales, les institutions carcérales et les publics, les représentations enracinées dans une culture tels que les mythes et les tabous sont confrontés à l'émergence locale de valorisations en interaction).
} 
Nous tenons ici à remercier tous les acteurs ayant accepté de prendre part à notre recherche, les membres des institutions muséales partenaires qui ont chaleureusement accueilli notre projet, les personnes qui nous ont donné l'autorisation de réaliser les enregistrements audiovisuels des visites et les institutions qui ont participé financièrement à la réalisation des différentes missions.

${ }^{3}$ À ce propos, il faut mentionner ici que, si la sémiotique des interactions telle qu'elle est développée par Eric Landowski (2006) constitue une assise théorique convaincante pour la caractérisation des modalités des régimes d'ajustement intersubjectif, elle ne nous semble pas permettre d'apporter des pièces probantes sur le plan des tensions entre (i) des processus de cogestion des pratiques langagières en interaction de manière multimodale et (ii) des interactions sémiotiques qui se réalisent entre différents systèmes de significations et de valorisations socialement partagés, du fait de l'absence d'attestation des interactions étudiées. Les enregistrements (audio)visuels constituent une source nécessaire, en ce qu'ils offrent un accès privilégié à la multimodalité des pratiques langagières - que nous appréhendons comme articulation entre (i) la dimension polysensorielle de l'expérience incarnée d'un environnement et (ii) le caractère polysémiotique des espaces, où cohabitent notamment des formes architecturales, linguistiques et picturales. L'un des enjeux d'une sémiotique des pratiques interactionnelles est alors de problématiser la réélaboration du sens de l'activité in situ à partir de la documentation produite par nous-mêmes.

${ }^{4}$ Une étude sémiotique a interrogé ces liens matériels et symboliques (Thiburce, 2018) en situant la place accordée à la caméra en tant qu'outil de recherche dans diverses méthodes, telles que celle de Michael Lynch (2002) qui a problématisé l'articulation entre données d'une anthropologie filmée des interactions (Lallier, 2009) et d'une sociologie des dispositifs (Licoppe, 2012).

${ }^{5}$ D'une part, on trouve une diversité de modèle d'objets techniques, telles que les caméras avec capot de visualisation intégré, les caméras $360^{\circ}$, des caméras avec dispositif d'eye-tracking. D'autre part, les potentialités d'un dispositif sont toujours remises en jeu, en ce qu'elles émergent des manières dont on s'en saisit en pratique et en fonction des lieux où on le mobilise.

${ }^{6}$ Sur la page du site internet adressée aux publics lycéens («accueil» > «enseignants»> «lycées »):

Cette visite couplée de l'exposition temporaire Prison, au-delà des murs du musée des Confluences et du Mémorial national de la prison de Montluc est un format unique de médiation pour mieux appréhender des questions de société à travers le milieu carcéral. La prison ainsi exposée permet d'interroger les consciences à travers des thématiques fortes. Le parcours de la prison de Montluc offrira un regard plus historique grâce à la visite du Mémorial et de l'ouverture exceptionnelle de l'aile des femmes, plus contemporaine, utilisée jusqu'en 2009.

${ }^{7}$ La structure de ces ateliers a été décrite dans une autre participation au CMLF 2020 intitulée " C'est nous qui punissons. "Quels enjeux d'un discours muséal sur les prisons?.

${ }^{8}$ On peut notamment mentionner le cas de la visite du manoir de Kerazan en Bretagne disponible dans la base de données CLAPI : http://clapi.ish-lyon.cnrs.fr/V3 Feuilleter.php?num corpus=113

${ }^{9}$ Nous avions déjà utilisé un tel dispositif dans une recherche passée. Pour une question d'anonymisation de notre article, nous y ferons référence dans une version ultérieure du texte.

${ }^{10}$ Voir le site de la CCC de l'UMR 5191 ICAR : http://icar.cnrs.fr/ccc/

${ }^{11}$ Dans la perspective d'une sémiotique des pratiques, Jacques Fontanille (2008: 77) a notamment problématisé la perméabilité des niveaux de pertinence sémiotique (signes, textes, objets, pratiques, stratégies/tactiques, formes de vie) en mettant en regard une «mise à plat » d'une scène pratique à partir de la schématisation opérée par un pictogramme et une «mise en relief» de cette scène à partir de la reconstitution de l'interaction présupposée par ce pictogramme.

${ }^{12}$ On adopte ici les conventions constituées par le groupe ICOR et mobilisées plus largement en analyse des interactions :

lyon2.fr/projets/corinte/documents/2013_Conv_ICOR_250313.pdf

Aussi, dans la phase de traitement du signal sonore et dans la réalisation des transcriptions, nous assurons une anonymisation des données relatives aux participants. De plus, avant chaque enregistrement, nous avons contacté l'ensemble des participants et, le cas échéant, de leur représentant légal, de manière (i) à les informer de notre présence le jour de l'activité à laquelle ils allaient prendre part et (ii) à nous assurer d'avoir leur autorisation pour la réalisation et la diffusion des enregistrements à des fins de recherche.

${ }^{13}$ Contrairement aux enregistrements que produiraient des documentaristes, ces données ne sauraient être diffusées dans l'espace social de manière autonome à un discours descriptif et/ou analytique. 


\footnotetext{
${ }^{14}$ Dans le champ de l'analyse des interactions, une collection est définie comme « a set of fragments, then, to explicate a single phenomenon or a single domain of phenomena ». Elle est à différencier d'une single case analysis consistant en l'analyse d'une occurrence particulière, où «resources of past work on a range of phenomena and organizational domains in talk-in-interaction are brought to bear on the analytic explication of a single fragment of talk », selon Schegloff (1987 : 101) cité par Lorenza Mondada (2008). Ici, le terme de collection est à appréhender dans un sens plus large, en tant que constellation de cas permettant d'illustrer la mobilisation et la thématisation du dispositif d'enregistrement (caméras, micros, présence de l'enquêteur sur le terrain, etc.) par les participants.

${ }^{15}$ Source : The Language Archive, Max Planck Institute, for Psycholinguistics https://tla.mpi.nl/tools/tla-tools/elan
} 